\title{
Minimal Residual Disease in Multiple Myeloma
}

\author{
Nikhil C. Munshi, VA Boston Healthcare System, Jerome Lipper Multiple Myeloma Center, Dana-Farber Cancer Institute, \\ Harvard Medical School, Boston, MA \\ Kenneth C. Anderson, Jerome Lipper Multiple Myeloma Center, Dana-Farber Cancer Institute, Harvard Medical School, \\ Boston, MA
}

See accompanying article on page 2540

Progress in the treatment of multiple myeloma has increased extent and frequency of response, as well as prolonged progressionfree (PFS) and overall survival (OS). Randomized trials of high-dose therapy and autologous stem cell transplantation in the 1990s showed the latter to be superior to standard dose therapy and for the first time achieved complete responses. ${ }^{1,2}$ The advent of novel therapies in the past decade has further transformed treatment. In particular, immunomodulatory drugs (thalidomide/lenalidomide/pomalidomide) and the proteasome inhibitors (bortezomib, carfilzomib), when combined as initial therapy for newly diagnosed patients, can achieve universal responses and complete responses (CRs) in a significant fraction of patients. ${ }^{3}$ Finally, the use of consolidation and maintenance therapy with novel agents can now further increase CR rate and prolong duration of response. ${ }^{4-7}$

Along with these improvements in therapeutic strategy, the definition of CR has evolved over time. Studies 30 years ago, before the advent of transplantation, defined CR as greater than $75 \%$ reduction in myeloma paraprotein, which was achieved in only a small fraction of patients. With high-dose therapy significant cytoreduction was achieved, and the definition of CR evolved to include not only disappearance of clonal plasma cells in bone marrow, but also absence of paraprotein in urine and serum by immunofixation, which was achieved in up to $30 \%$ patients. Stringent CR, as more recently defined by the International Myeloma Workshop, ${ }^{8}$ includes these parameters along with a normal kappa:lambda free light chain ratio. However, the fact that all patients achieving CR, as defined in this way, go on to experience relapse suggests that clinically meaningful residual disease is not detectable by these parameters. Molecular complete responses (mCR), defined as absence of detectable disease by polymerase chain reaction for Ig gene rearrangement, was until recently observed only in a fraction of patients undergoing allogeneic transplantation and was associated with prolonged PFS and OS. ${ }^{910}$ These allogeneic transplantation studies suggested the clinical importance of achieving mCR, but this extent of response was not achievable in the autologous setting.

The incorporation of novel therapies into the initial management of newly diagnosed myeloma has transformed therapy, with increased frequency and extent of response. It was therefore necessary to develop reproducible sensitive assays for detecting and monitoring minimal residual disease $(\mathrm{MRD})$ and to define its prognostic value in predicting for PFS and OS, to allow for informing consolidation and maintenance strategies, and to evaluate the comparative efficacy of novel therapies. These methods include allele-specific oligonucleotide PCR (ASO-PCR) capable of detecting up to one clonal cell in $10^{5}$ normal cells and immunophenotypic assays detecting one clonal cell in $10^{4}$ normal cells by use of $\geq$ seven-color multiparameter flow cytometry $(\mathrm{MPF})^{11-13}$ Although the ASO-PCR method may provide greater sensitivity, it remains a difficult assay to be performed, as it requires the generation of patient-specific primers. The advantages of MPF include the ready ability to perform the assay, as well as short turnaround time. In MPF, quantitating residual myeloma cells requires sophisticated analysis but is automated to promptly obtain objective results. ${ }^{11,14,15} \mathrm{~A}$ comparison of $\mathrm{CR}$ detection by negative immunofixation $(\mathrm{CR})$, normal serum free light chain ratio $(\mathrm{sCR})$, and undetectable myeloma cells by MPF (immunophenotyping CR - iCR) in 102 patients with multiple myeloma treated with novel agents showed that $43 \%$ patients achieved CR, 30\% achieved sCR, and 30\% achieved $\mathrm{iCR}$. There was no significant survival difference between patients with sCR versus CR; importantly, however, patients in iCR showed significantly increased PFS and time to progression (TTP) compared with those in sCR or CR, suggesting increased sensitivity of MPF to detect MRD. ${ }^{16}$ Although a head to head comparison between iCR assessed by MPF and mCR measured by ASO-PCR showed that ASO-PCR is slightly more sensitive and specific than MPF, it was applicable in a lower proportion of MM patients ( $75 \%$ versus $90 \%$, respectively) and more time-consuming than MPF. ${ }^{17}$ Interestingly, in this study ASO-PCR and MPF were able to detect residual myeloma cells in 17 and 11 patients, respectively. Progression-free survival for those patients without versus with MRD detected by ASO-PCR was 34 versus 15 months, respectively $(P=.04)$ and by MPF was 27 versus 10 months, respectively $(P=.05)$. More recently, a novel sequencing-based method has been developed to quantify cells with specific molecular signatures. This method identifies clonal gene rearrangements in diagnostic samples using consensus primers to universally amplify rearranged IgH and $\mathrm{k}$ gene segments, followed by high-throughput sequencing and informatic algorithms to then quantify these rearrangements in follow-up MRD samples. Compared to the ASO-PCR method, this new assay does not require patient-specific customization, which improves scalability and reduces $\operatorname{costs}^{18} \mathrm{~A}$ comparison using such newer sequencing technologies with high resolution MPF is required to determine the relative specificity and sensitivity of these assays to detect $\mathrm{MRD}$, as well as their relative ability to identify infrequent tumor clones. 


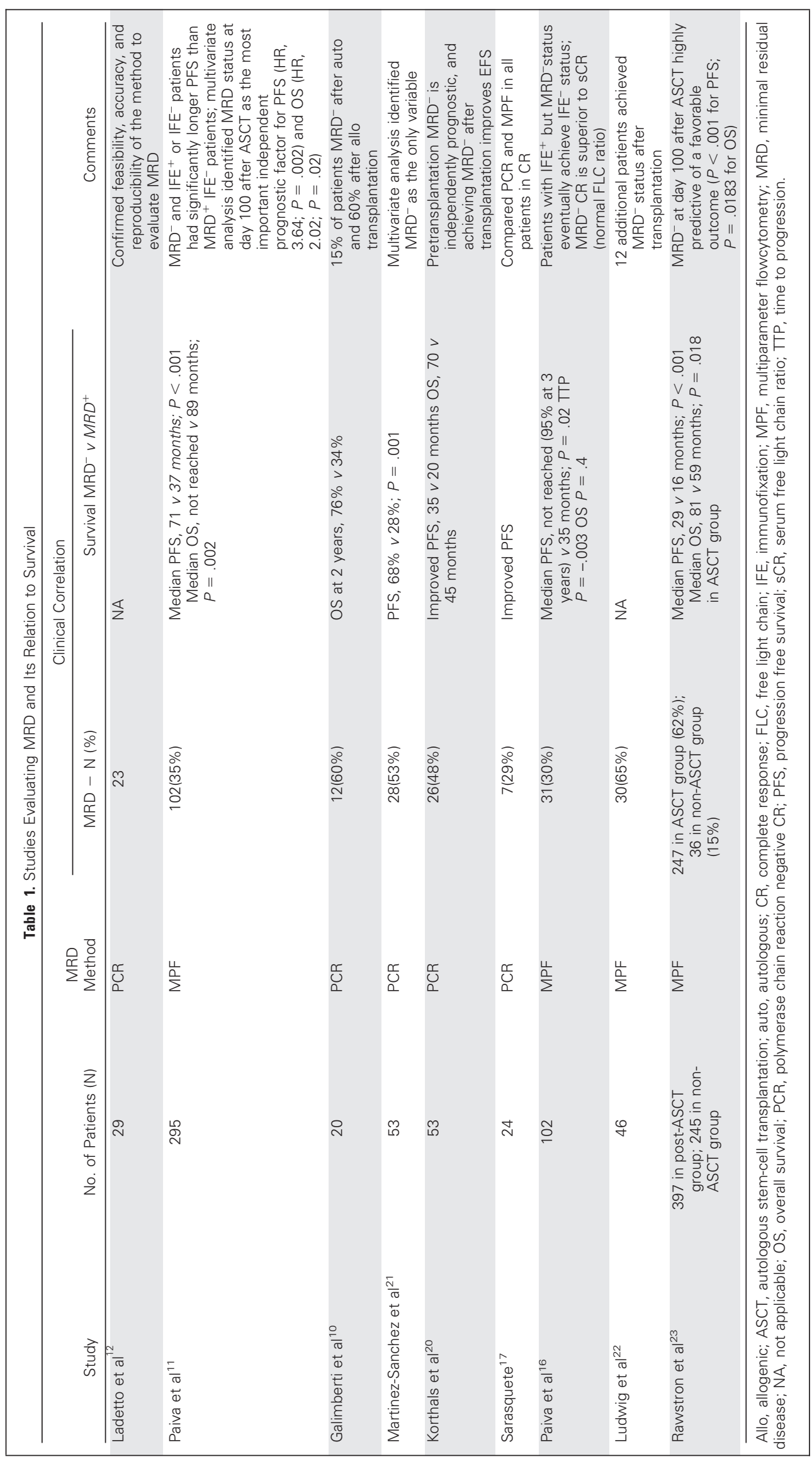


A critical question now is whether this ability to detect MRD by MPF or ASO-PCR has clinical implications for PFS and OS, and whether it may inform therapy and allow us to tailor therapeutic decisions. For example, Paiva et $\mathrm{al}^{19}$ investigated prognostic markers able to predict unsustained CR in a series of 241 patients in CR at day +100 after high-dose therapy/autologous stem-cell transplantation (HDT/ASCT). Besides the presence of baseline high-risk cytogenetics by fluorescent in situ hybridization (hazard ratio 17.3; $P=.002$ ), persistent MRD by MPF at day +100 after HDT/ASCT (hazard ratio $8.0 ; P=.005)$ was the only other independent factors that predicted for unsustained CR and a poor outcome, evidenced by a median 39-month OS. Using ASO-PCR, Korthals et $\mathrm{al}^{20}$ identified 26 as lowand 27 as high-MRD patients after HDT/ASCT: the low-MRD group compared to high-MRD group had significantly longer median EFS (35 $v 20$ months, respectively; $P=.001)$ and OS (70 $v 45$ months, respectively; $P=.04$ ). Other studies identifying MRD status using ASO-PCR or MPF and correlating with clinical outcomes are detailed in Table 1.

In the article that accompanies this editorial, Rawstron et $\mathrm{al}^{23}$ assessed MRD using MPF in a large cohort of uniformly treated patients following induction and at 100 days post ASCT, as well as postinduction therapy in a nontransplantation group. This study demonstrates the applicability and clinical utility of assessing MRD. It confirms the feasibility of carrying out MRD analysis in a large majority of patients enrolled in a multicenter study and demonstrates clinical utility of this assay, given that patients with MRD had inferior outcome compared to those without detectable MRD. Importantly, this study also showed that maintenance therapy provides benefit to those patients who are MRD negative; moreover, maintenance therapy can convert patients to MRD negative status, which correlates with improved outcome. One limitation of this and other studies using ASO-PCR and MPF assays of MRD is their inability to detect extramedullary disease, which is now being observed more frequently at time of relapse after novel therapies. PET/CT scanning, as pioneered in Arkansas, ${ }^{24}$ Bologna, ${ }^{25}$ and by other investigators, ${ }^{26,27}$ may add sensitivity for detection of both extramedullary and medullary MRD.

The current and other studies therefore highlight that MRDnegative status can be achieved in the era of novel agents and is predictive of superior outcome. If validated in future studies, MRD may serve as a biomarker to inform therapy and as a surrogate for OS. Specifically, achieving MRD-negative status may become a goal of future studies using induction, transplantation, consolidation, and/or maintenance therapies. With available technologies and ease of MRD measurement, it is now time to carry out additional large prospective studies to define the clinical significance of MRD and its impact on patient outcome in myeloma. It may be possible, as in chronic myeloid leukemia, to both assess and monitor MRD using standardized assays and thereby both inform therapy and improve patient outcome.

\section{AUTHORS' DISCLOSURES OF POTENTIAL CONFLICTS OF INTEREST}

Although all authors completed the disclosure declaration, the following author(s) and/or an author's immediate family member(s) indicated a financial or other interest that is relevant to the subject matter under consideration in this article. Certain relationships marked with a " $U$ " are those for which no compensation was received; those relationships marked with a " $C$ " were compensated. For a detailed description of the disclosure categories, or for more information about ASCO's conflict of interest policy, please refer to the Author Disclosure Declaration and the Disclosures of Potential Conflicts of Interest section in Information for Contributors. Employment or Leadership Position: None Consultant or Advisory Role: Nikhil C. Munshi, Celgene (C), Onyx Pharmaceuticals (C), Merck (C); Kenneth C. Anderson, Celgene (C), Onyx Pharmaceuticals (C), sanofi-aventis (C), Gilead Sciences (C) Stock Ownership: Nikhil C. Munshi, Oncopep; Kenneth C. Anderson, Acetylon, Oncopep

Honoraria: None Research Funding: None Expert Testimony: None Other Remuneration: None

\section{AUTHOR CONTRIBUTIONS}

Manuscript writing: All authors

Final approval of manuscript: All authors

\section{REFERENCES}

1. Mahindra A, Laubach $\mathrm{J}$, Raje $\mathrm{N}$, et al: Latest advances and current challenges in the treatment of multiple myeloma. Nat Rev Clin Oncol 9:135-143, 2012

2. Palumbo A, Anderson K: Multiple myeloma. N Engl J Med 364:1046-1060, 2011

3. Anderson $\mathrm{KC}$ : Oncogenomics to target myeloma in the bone marrow microenvironment. Clin Cancer Res 17:1225-1233, 2011

4. Badros AZ: Lenalidomide in myeloma-a high-maintenance friend. N Engl J Med 366:1836-1838, 2012

5. Attal M, Lauwers-Cances $V$, Marit G, et al: Lenalidomide maintenance after stem-cell transplantation for multiple myeloma. N Engl J Med 366:1782-1791, 2012

6. McCarthy PL, Owzar K, Hofmeister CC, et al: Lenalidomide after stem-cell transplantation for multiple myeloma. N Engl J Med 366:1770-1781, 2012

7. Palumbo A, Hajek R, Delforge $M$, et al: Continuous lenalidomide treatment for newly diagnosed multiple myeloma. N Engl J Med 366:1759-1769, 2012

8. Munshi NC, Anderson KC, Bergsagel PL, et al: Consensus recommendations for risk stratification in multiple myeloma: Report of the International Myeloma Workshop Consensus Panel 2. Blood 117:4696-4700, 2011

9. Martinelli G, Terragna C, Zamagni E, et al: Molecular remission after allogeneic or autologous transplantation of hematopoietic stem cells for multiple myeloma. J Clin Oncol 18:2273-2281, 2000

10. Galimberti S, Benedetti E, Morabito F, et al: Prognostic role of minimal residual disease in multiple myeloma patients after non-myeloablative allogeneic transplantation. Leuk Res 29:961-966, 2005

11. Paiva B, Vidriales MB, Cervero J, et al: Multiparameter flow cytometric remission is the most relevant prognostic factor for multiple myeloma patients who undergo autologous stem cell transplantation. Blood 112:40174023, 2008

12. Ladetto $M$, Donovan JW, Harig $S$, et al: Real-time polymerase chain reaction of immunoglobulin rearrangements for quantitative evaluation of minimal residual disease in multiple myeloma. Biol Blood Marrow Transplant 6:241253, 2000

13. Garcia-Sanz R, Lopez-Perez R, Langerak AW, et al: Heteroduplex PCR analysis of rearranged immunoglobulin genes for clonality assessment in multiple myeloma. Haematologica 84:328-335, 1999

14. Paiva $B$, Vidriales MB, Perez JJ, et al: Multiparameter flow cytometry quantification of bone marrow plasma cells at diagnosis provides more prognostic information than morphological assessment in myeloma patients. Haematologica 94:1599-1602, 2009

15. Rawstron AC, Orfao A, Beksac M, et al: Report of the European Myeloma Network on multiparametric flow cytometry in multiple myeloma and related disorders. Haematologica 93:431-438, 2008

16. Paiva B, Martinez-Lopez J, Vidriales MB, et al: Comparison of immunofixation, serum free light chain, and immunophenotyping for response evaluation and prognostication in multiple myeloma. J Clin Oncol 29:1627-1633, 2011

17. Sarasquete ME, Garcia-Sanz R, Gonzalez D, et al: Minimal residual disease monitoring in multiple myeloma: A comparison between allelic-specific oligonucleotide real-time quantitative polymerase chain reaction and flow cytometry. Haematologica 90:1365-1372, 2005

18. Faham M, Zheng J, Moorhead M, et al: Deep-sequencing approach for minimal residual disease detection in acute lymphoblastic leukemia. Blood 120:5173-5180, 2012

19. Paiva B, Gutierrez NC, Rosinol L, et al: High-risk cytogenetics and persistent minimal residual disease by multiparameter flow cytometry predict unsustained complete response after autologous stem cell transplantation in multiple myeloma. Blood 119:687-691, 2012 
20. Korthals $M$, Sehnke $N$, Kronenwett $R$, et al: The level of minimal residual disease in the bone marrow of patients with multiple myeloma before high-dose therapy and autologous blood stem cell transplantation is an independent predictive parameter. Biol Blood Marrow Transplant 18:423-431 e3, 2012

21. Martinez-Sanchez $P$, Montejano $L$, Sarasquete ME, et al: Evaluation of minimal residual disease in multiple myeloma patients by fluorescentpolymerase chain reaction: The prognostic impact of achieving molecular response. Br J Haematol 142:766-774, 2008

22. Ludwig $H$, Viterbo $L$, Greil $R$, et al: Randomized phase II study of bortezomib, thalidomide, and dexamethasone with or without cyclophosphamide as induction therapy in previously untreated multiple myeloma. J Clin Oncol 31:247-255, 2013

23. Rawstron AC, Child JA, de Tute RM, et al: Minimal residual disease assessed by multiparameter flow cytometry in multiple myeloma: Impact on outcome in the Medical Research Council Myeloma IX study. J Clin Oncol $31: 2540-2547,2013$
24. Bartel TB, Haessler J, Brown TL, et al: F18-fluorodeoxyglucose positron emission tomography in the context of other imaging techniques and prognostic factors in multiple myeloma. Blood 114:2068-2076, 2009

25. Zamagni E, Patriarca F, Nanni C, et al: Prognostic relevance of 18-F FDG PET/CT in newly diagnosed multiple myeloma patients treated with up-front autologous transplantation. Blood 118:5989-5995, 2011

26. Tan $E$, Weiss BM, Mena $E$, et al: Current and future imaging modalities for multiple myeloma and its precursor states. Leuk Lymphoma 52:16301640, 2011

27. Walker RC, Brown TL, Jones-Jackson LB, et al: Imaging of multiple myeloma and related plasma cell dyscrasias. J Nucl Med 53:1091-1101, 2012

DOI: $10.1200 / J C O .2013 .49 .2124$; published online ahead of print at www.jco.org on June 3, 2013

\section{Help Your Patients Understand Advanced Cancer Care Planning}

ASCO's Advanced Cancer Care Planning booklet is designed to help people with advanced cancer and their families and caregivers understand the diagnosis and treatment options for advanced cancer, discuss these options for care throughout the course of the illness, and find support.

Download the booklet in English and Spanish at cancer.net/advancedcancer or order copies for your practice at cancer.net/estore. 


\section{Acknowledgment}

Supported in part by Grants No. I01-BX001584 from the Veterans' Administration and RO1-124929 from the National Institutes of Health (N.C.M); Grants No. P50-100007, PO1-78378, and PO1-155258 from the National Institutes of Health (N.C.M and K.C.A.); and Grant No. RO1-50947 from the National Institutes of Health (K.C.A). K.C.A. is American Cancer Society Professor in Oncology. 